\title{
Contribution of Artisanal and Small-Scale Mining to Sustainable Territorial Development through Strengthening Capabilities of Mining Communities
}

\author{
Gustavo A. Aristizábal-H*, Oscar J. Restrepo-B and J. Alejandro Delgado-J \\ Materials and Minerals Department, School of Mines, Universidad Nacional de Colombia, Medellín
}

\begin{abstract}
In Antioquia region of Colombia - South America most of extractive activity is developed as artisanal and smallscale level, exploiting different types of minerals resources but predominantly gold. This is not only an ancestral livelihood of hundreds of families in this region, but also their unique way of subsistence. However, that situation has not had a positive effect in the sustainable development of this territory because of several unsustainability factors mainly related to mercury use for gold extraction, the incorrect management of tailings and the informal way in which miners perform their activity. In 2010 Antioquia was the place with the highest contamination by mercury in the world with serious effects in ecosystems and health of habitants of this region (Veiga 2010)

This paper presents a Socio-Technical Model focused on strengthening capabilities and social capital of artisanal and small-scale miners and its surrounding communities in Antioquia region. This is based on processes of evaluation, design and implementation of appropriate technologies to obtain "clean minerals" without using mercury and with a right management of tailings. In gold extraction it is about gravitational methods of concentration, which take advantage of high density of this element. These methods not only are cleaner but more profitable for miners.

Notwithstanding its technical origins, the current model proposes a holistic framework that manages the elimination of mercury in mining not only as a technology transfer matter. Thus, more than formalization the strengthening process seeks to promote a business vision in miners and to develop productive chains in surrounding communities, this means to improve the skills of artisanal and small-scale miners to organize in a corporative way and to help surrounding communities to provide support and supply services to mining and even to other companies. Besides, the articulation with local institutions drives to reinforce their functions of control and accompaniment. In shorthand, this effort aims to empower local institutions, miners and surrounding communities in such a way they are able to face together their own development challenges in a sustainable way.
\end{abstract}

Keywords: artisanal and small scale miners, sustainable development, clean technologies, surrounding communities, productive chains, local institutions.

\section{Introduction}

This work is about socio-technical intervention of artisanal and small-scale gold mining units (ASGMU) in the Antioquia region in Colombia. It has been developed mainly through two stages during the last four years by CIMEX Minerals Institute (CMI), School of Mines, Universidad Nacional de Colombia in Medellin that is a multidisciplinary team dedicated to mining activity since 1983. However, there was a preliminary wide effort in which the CMI contributed. This was called Colombia Mercury Project.

In Antioquia region most of extractive activity is developed as artisanal and small-scale mining (ASM) level, exploiting different types of minerals resources but predominantly gold. Most of this initiative has been developed in the Northeast of Antioquia where ASM represents $95 \%$ of extractive labor with near to 11,600 people working on it.
However, this livelihood has not had a positive effect in the sustainable development of this territory largely because of four unsustainability factors:

1) Contamination of ecosystems due to mercury use for gold extraction and incorrect management of tailings.

2) Low productivity of ASM due to the use of inefficient and health risky methods of extraction.

3) The informal way in which miners develop their extractive activities.

4) The weakening of social capital of communities as a result of conflicts for the access to natural resources, and from the connection that mercury trade keeps with guerrillas and other armed groups outside the law.

This project has been worked with many artisanal and small-scale miners in Antioquia through the application of a Socio-Technical Model (STM) focused on strength their

* Corresponding Author: A. Gustavo-A, email: gaaristizabalh@unal.edu.co

Copyright @ 2017 Canamaple Academia Services, http://press.camdemia.ca

DOI: 10.15273 /gree.2017.02.036 
capabilities and technical competences and reinforce the social capital of mining communities. To achieve a balance in small-scale mining activities, a business culture among miners must be fostered and capabilities must be developed that lead to more resilient and entrepreneurial thinking models, helping mining communities to overcome behaviors that prevent them from interacting and collaborating between them and with other external actors.

The more isolated an individual or a community, the more difficult to them to collaborate. Consequently, strengthening the links between miners and of them with their stakeholders is a main focus of the STM to strengthen their partnership skills and the ability to form networks and productive linkages between themselves and with other external actors.

Technical competences of STM are based on processes of evaluation, design and implementation of appropriate technologies to obtain "clean minerals" without using mercury and with a right management of tailings. In gold mining it is about gravitational methods of classification and concentration, which take advantage of high density of this element.

\section{Stages of the Initiative}

Firstly, CMI participated in the Colombia Mercury Project (2011 - 2012) of United Nations Industrial Development Organization (UNIDO) with the collaboration of Environmental Agency of Antioquia -CORANTIOQUIA-, The Mining Office of the Government of Antioquia and the University of British Columbia from Canada. Later, a second effort was driven by CMI (2013 - 2014) with the financing support of Colombian Ministry of Environment and CORANTIOQUIA.

The third stage is currently in course and should be highlighted because it has not only the institutional supports at local level but also -for first time in this kind of projects in Antioquia- with a formal engagement with an artisanal and small-scale miners association. This is a great opportunity in the purpose of reinforcing social capital of communities.

\section{Direct and Indirect Beneficiaries of This Initiative}

The direct beneficiaries of this effort are mining communities and neighbouring ones due to productive chains opportunities. Otherwise, the articulation with local institutions drives to strengthening their functions of control and accompaniment. An efficient and sustainable mining protects ecosystems, diversify and stimulate the local economy through production chains and economic diversification. For example, in sites where a more profitable ASM has been reached, it has been seen miners hiring external services such as plumbing and provision of food that are supplied by neighbouring families.

Considering that illegal mining in Colombia has become a source of funding for armed groups outside the law, the reduction of mercury use in ASM also contributes to the process of peace that Colombia is presently advancing to finish the last conflict in the western hemisphere, since this initiative takes this communities away from the illegality that still surrounds gold industry and mercury trade in Colombia.

\section{Features of the Project}

Clean technologies through gravitational processes in mining are not a quite fresh innovation. However, many initiatives for its incorporation in ASM tend to fail due to they have not consider socio-economic variables.

This project proposes a more holistic framework, due to addresses "the elimination of mercury in mining" not only as a technology transfer matter. By this way and with a wide participation of communities, the achievements of the project have tend to sustained -even afterwards of CMI presence in sub region-, because miners have enhanced their proficiency to do their job less risky for their health, cleaner for the environment and more profitable.

Nonetheless, the complexity of challenges that ASM have to face leads to wide participation of people is not enough to accomplish the long term sustainability of goals reached, considering the institutional weakness in regions and the individualistic culture that tend to prevail in mining communities.

The core of this initiative is based on the principle that the challenges of sustainable development should not be addressed in isolation. Consequently, the STM seeks to strength municipalities in their control functions and improve the skills of artisanal and small-scale miners to establish and maintain partnerships with other actors in the territory. In few words, this effort aims to empower local institutions and mining communities in such a way they are able to face together their own development challenges in a sustainable mode.

\section{Business Case and Contribution to Sustainable Development}

Mining in Antioquia is the ancestral livelihood of hundreds of families and their unique way of subsistence. Nevertheless, the rudimentary conditions in which ASM is usually developed in this region not only make this activity inefficient, but also lead to negative impacts on the ecosystems, the health of miners and surrounding populations.

Besides to the several environmental and social contributions already mentioned, and in addition to the economic local perspective mentioned in item 3, clean gold mining technologies have demonstrated an increase in production efficiency of $80 \%$ over traditional mercurybased technologies. This improves the ASM business profitability and avoids economic sanctions by the environmental agency.

Despite multiple advantages of these technologies, miners are often reluctant to adopt them due to socioeconomic and cultural factors. Hence, the STM not only comprises continuous accompaniment with pilot demonstrations that show its efficiency (Photo 1), but also request the logistic and economic participation of mining communities in the implementation. 
Moreover, they contribute with almost $25 \%$ of equipment total costs, its transportation and assembly in ASGMU. This financial format of model makes people feel involved, and distances it from any assistentialism sense.

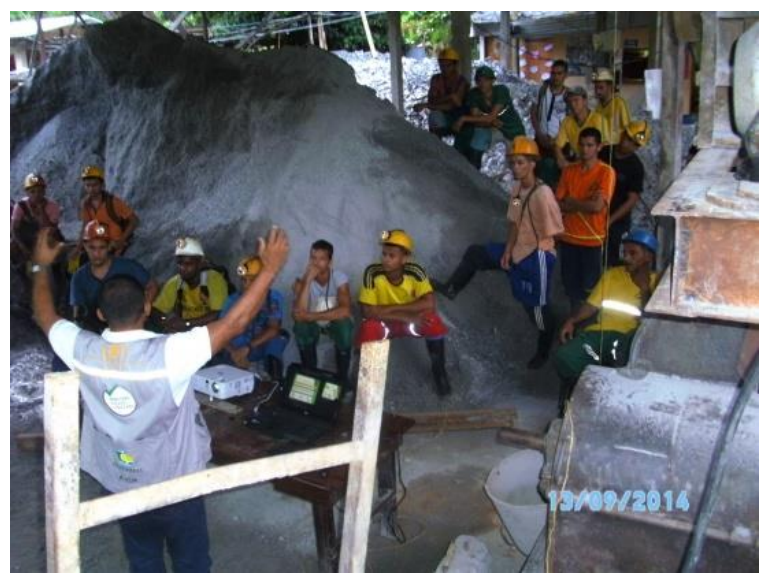

Photo 1. Pilot demonstrations to small-scale miners in the northeast of Antioquia region in Colombia.

STM seeks to strengthen the capabilities and social capital of miners, their neighbouring communities and local governments in order to boost territories and promote their sustainable development. At the same time, these processes provide information to the model that allows its improvement (Figure 1).

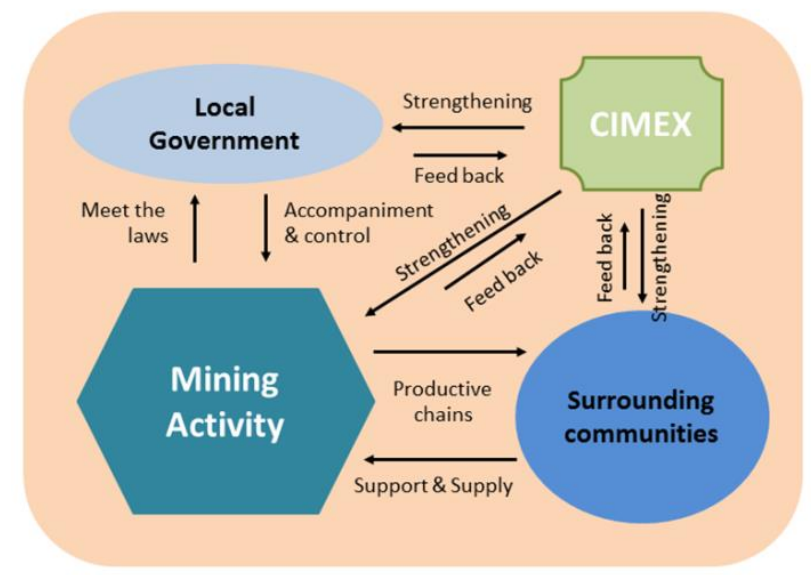

Figure 1. Socio-technical model applied to territory with artisanal and small scale mining activity.

\section{Some Results}

The purpose of Colombia Mercury Project was to improve the activities of ASGMU taking into account that five municipalities in Northeast of Antioquia with a population of 162,000 inhabitants were the world's largest mercury polluter in 2010, emitting 92 tons of mercury per year (Cordy et al 2011). The actions consisted of mercury losses assessment, health monitoring and awareness campaign.

This was supported by visits and technical demonstrations to 323 ASGMU.
As a result of this, 39 ASGMU were installed in Antioquia and mercury entering to the environment was reduced in $63 \%$ than in 2010. Accordingly, between 46 and 70 tons per year of mercury has been prevented from polluting the environment. The miners also realized that cleaner processes are more efficient than traditional ones (García et al 2015).

For a second stage, CMI selected 21 ASGMU in Antioquia to be intervened. With the modifications made in six of these ASGMU was possible to decrease the use of mercury by $50 \%$ (from $20-22$ pounds to $10-11$ pounds in amalgamation processes). Furthermore, an increase in production efficiency of 20\% was obtained (CIMEX 2014)

\section{Conclusions}

Whereas in the Northeast of Antioquia gold has been mined since pre-colonial times, other areas of this region have been agricultural -mainly coffee plantations- with lower presence of extractive activity. Nevertheless, during the last years hundreds of families have found in mining a subsistence alternative. This scenario has increased the ASM incidence in zones such as the Southwest of Antioquia, but reproducing the unsustainable behaviors from the northeastern (Photo 2).

Thus, in the current third stage of the project CMI established a formal alliance with the Miners Association of Southwest of Antioquia (ASOMISURA, in Spanish) with the purpose of replicate the MST. This joint work is oriented to position the ASM as a sustainable and alternative livelihood for this territory, to prevent environmental degradation and social conflicts, especially because the southwest of Antioquia still has no significant presence of armed groups outside the law.

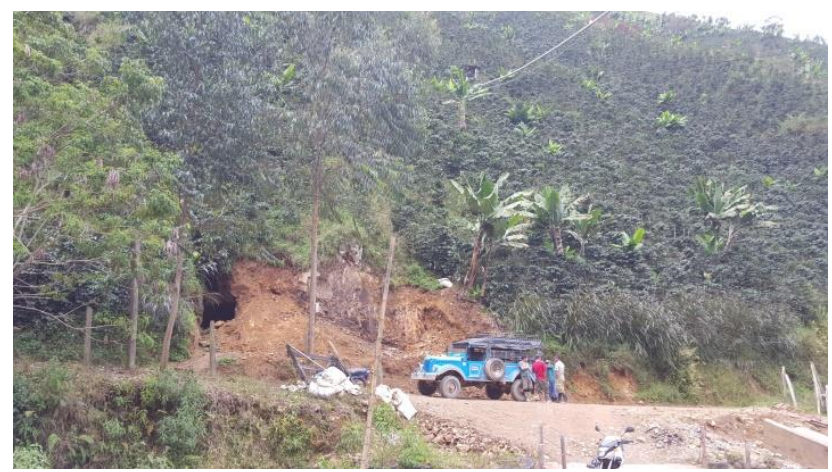

Photo 2. Artisanal gold mine under coffee plantations in the south-west of Antioquia region in Colombia.

In Colombian case, ASM also represents the $96 \%$ of its mining activity. Hence, although Antioquia region has the highest presence of ASM in the country, in fact this is present in 20 of its 32 regions and with under similar socio-technical conditions.

Notwithstanding, negative impacts due to ASM is not an exclusive phenomenon of Colombia. The International Labour Organization estimated more than 13 million 
artisanal and small scale miners working around the world in the beginning of the 2000s. In Latin America, Africa and Asia; ASM is a significantly economic activity. For instance, in Ghana the proportion of gold ASM increased from 6\% in 2000 to $23 \%$ in 2010 (Basu et al 2015), and is estimated that Brazil has 200.000 people dedicated to gold ASM (Cremers et al 2013).

The STM is a flexible tool and in continuous improvement focused on the evaluation of the socioeconomic previous conditions of territories in order to adjust its intervention processes. This adoption of appropriate technologies makes the project replicable in other regions from low- and middle-income countries.

Important contributions have been done through this project towards the implementation in Antioquia of clean ASM. However, both laboratory and ASGMU pilot tests have demonstrated that $100 \%$ of mercury reduction simultaneously with an $80 \%$ of increasing on the production efficiency is something achievable. The obstacles to overcome in order to accomplish these figures are mainly related to the economic limitations of mining families, difficult access to many ASGMU -no roads- and the cultural inertia of communities.

This experience has shown us that these difficulties are often solved through associative work. Therefore, the reinforcement of social capital of mining communities is an inescapable issue.

\section{References}

Basu, N., E. Clarke, A. Green, B. Calys-T, L. Chan, M. Dzodzomenyo, J. Fobil, R.N. Long, R.L. Neitzl, S. Obiri, E. Odei, L. Ovadje, R. Quansah, M. Rajaee and M.L. Wilson, 2015. Integrated assessment of artisanal and small-scale gold mining in Ghana - Part 1: human health review. International Journal of environmental research public health, 12(5): 5143 - 5176.

CIMEX, 2014. Desarrollar la implementación de tecnologías limpias en unidades mineras ubicadas en las zonas auríferas del departamento de Antioquia. http://www.corantioquia.gov.co/sitios/ExtranetCoranti oquia/ciadoc/SUELO/GA_CV_1144_2013.pdf.

Cordy, P., M. Veiga, I. Salih, S. Al-Saadi, S. Console, O. García, L.A. Mesa, P.C. Velásquez-L and M. Roeser, 2011. Mercury contamination from artisanal gold mining in Antioquia, Colombia: The world's highest per capita mercury pollution, Science of the total Environment, s 410 - 411(411): 154 - 160.

Cremers, L., J. Kolen and M.D. Theije, 2013. Small-scale gold mining in the Amazon: The cases of Bolivia, Brazil, Colombia, Peru and Suriname. Cedla, 1 - 16.

García, O., M.M. Veiga, P. Cordy, O.E. Suescún, J.M. Molina and M. Roeser, 2015. Artisanal gold mining in Antioquia, Colombia: a successful case of mercury reduction. Journal of Cleaner Production, 90:244-252.

Veiga, M., 2010. Antioquia, Colombia: The world's most polluted place by mercury: impressions of two field trip, UNIDO. 